\title{
Online Signal Extraction by Robust Linear Regression
}

\author{
Ursula Gather $^{1}$, Karen Schettlinger ${ }^{1}$ and Roland Fried $^{2}$ \\ ${ }^{1}$ Department of Statistics, University of Dortmund, 44221 Dortmund, Germany \\ 2 Department of Statistics, University Carlos III, 28903 Getafe (Madrid), Spain
}

\begin{abstract}
In intensive care, time series of vital parameters have to be analysed online, i.e. without any time delay, since there may be serious consequences for the patient otherwise. Such time series show trends, slope changes and sudden level shifts, and they are overlaid by strong noise and many measurement artefacts. The development of update algorithms and the resulting increase in computational speed allows to apply robust regression techniques to moving time windows for online signal extraction. By simulations and applications we compare the performance of least median of squares, least trimmed squares, repeated median and deepest regression for online signal extraction.
\end{abstract}

Keywords: Robust filtering, least median of squares, least trimmed squares, repeated median, deepest regression, breakdown point.

\section{Introduction}

The online analysis of vital parameters in intensive care requires fast and reliable methods as a small fault can yield life-threatening consequences for the patient. Methods need to be able to deal with a high level of noise and measurement artefacts and provide robustness against outliers. The variables in question include for example heart rate, pulse, temperature and different blood pressures.

Davies, Fried and Gather (2004) apply robust regression techniques to moving time windows to extract a signal containing constant periods, monotonic trends with time-varying slopes and sudden level shifts. In this context, they 
compare $L_{1}$, repeated median $(R M)$, and least median of squares (LMS) regression. They report that repeated median regression is preferable to $L_{1}$ in most respects; opposed to these methods, LMS regression tends to instabilities and it is slower, but it traces level shifts better and it is less biased in the presence of many large outliers.

These findings concern the signal approximation in the centre of each time window, i.e. with some time delay. Since fast reaction is of utmost importance in intensive care, we exploit online versions of such procedures. Resuming the work from Davies et al. (2004) we compare four regression methods here:

In Rousseeuw, Van Aelst and Hubert (1999, p. 425), Rousseeuw points out that he considers LMS to be outperformed by least trimmed squares (LTS) regression because of its smoother objective function which results in a higher efficiency; the only advantage of $L M S$ would be its minimax bias among all residual-based estimators. It is of interest here whether LTS regression may outmatch $L M S$ with respect to stability. Additionally, we investigate deepest regression $(D R)$, which is expected to deal well with asymmetric and heteroscedastic errors (Rousseeuw and Hubert 1999) and compare it to $R M$ regression which showed best performance for delayed signal extraction.

Section 2 introduces the methods of interest and discusses some of their properties. In Section 3, a simulation study is carried out in order to investigate the performance of the methods in different data situations. Section 4 describes applications to some time series from intensive care, and finally, Section 5 closes with some concluding remarks.

\section{Procedures for Online Signal Extraction}

In the following, we consider a real valued time series $\left(\mathbf{y}_{t}\right)_{t \in \mathbb{Z}}$ observed at time points $t=1, \ldots, N$. For the applicability of robust regression methods, we assume the data to be locally well approximated by a linear trend. This means, within time windows of fixed length $n=2 m+1$ we assume a model

$$
y_{t+i}=\mu_{t}+\beta_{t} i+\varepsilon_{t, i}, \quad i=-m, \ldots, m,
$$


where $\mu_{t}$ denotes the underlying level of the signal and $\beta_{t}$ the slope at time $t$; $\varepsilon_{t, i}$ denote independent error terms with zero median. Below, we consider different distributional assumptions for $\varepsilon_{t, i}$.

Regarding only one time window, we may drop the index $t$ for simplicity. Hence, for a time window centred at time $t$ we write $y_{i}=\mu+\beta i+\varepsilon_{i}$ for $i=-m, \ldots, m$. The window width $n$ is chosen based on statistical and medical arguments as explained in Section 3.

\subsection{Methods for Robust Regression}

Let now $\mathbf{y}=\left(y_{-m}, \ldots, y_{m}\right)^{\prime}$ denote a time window of width $n$ from $\left(\mathbf{y}_{t}\right)_{t \in \mathbb{Z}}$, and let $r_{i}=y_{i}-(\tilde{\mu}+\tilde{\beta} i), i=-m, \ldots, m$, denote the corresponding residuals. For estimation of the level $\mu$ of the signal and the slope $\beta$ we consider the following robust regression functionals $T: \mathbb{R}^{n} \rightarrow \mathbb{R}^{2}$ :

1. Least Median of Squares (Rousseeuw 1984)

$$
T_{L M S}(\mathbf{y})=\left(\tilde{\mu}_{L M S}, \tilde{\beta}_{L M S}\right)=\arg \min _{\tilde{\mu}, \tilde{\beta}}\left\{\operatorname{med}\left(r_{i}^{2} ; i=-m, \ldots, m\right)\right\} .
$$

2. Least Trimmed Squares (Rousseeuw 1983)

$$
T_{L T S}(\mathbf{y})=\left(\tilde{\mu}_{L T S}, \tilde{\beta}_{L T S}\right)=\arg \min _{\tilde{\mu}, \tilde{\beta}} \sum_{k=1}^{h}\left(r^{2}\right)_{k: n},
$$

where $\left(r^{2}\right)_{k: n}$ denotes the $k$ th ordered squared residual for the current time window, i.e. $\quad\left(r^{2}\right)_{1: n} \leq \ldots \leq\left(r^{2}\right)_{k: n} \leq \ldots \leq\left(r^{2}\right)_{n: n}$ for any $k \in\{1, \ldots, n\}$, and $h$ is a trimming proportion. We take $h=\lfloor n / 2\rfloor+1$ below.

3. Repeated Median (Siegel 1982)

$$
\begin{aligned}
T_{R M}(\mathbf{y}) & =\left(\tilde{\mu}_{R M}, \tilde{\beta}_{R M}\right) \\
\text { with } \tilde{\beta}_{R M} & =\operatorname{med}_{i}\left(\operatorname{med}_{j \neq i} \frac{y_{i}-y_{j}}{i-j} ; i, j=-m, \ldots, m\right) \\
\text { and } \tilde{\mu}_{R M} & =\operatorname{med}_{i}\left(y_{i}-\tilde{\beta}_{R M} i ; \quad i=-m, \ldots, m\right),
\end{aligned}
$$

where the median for an even sample size is defined as the mean of the two midmost observations. 
4. Deepest Regression (Rousseeuw and Hubert 1999)

$$
T_{D R}(\mathbf{y})=\left(\tilde{\mu}_{D R}, \tilde{\beta}_{D R}\right)=\arg \max _{\tilde{\mu}, \tilde{\beta}}\{\operatorname{rdepth}((\tilde{\mu}, \tilde{\beta}), \mathbf{y})\},
$$

where the regression depth of a fit $(\tilde{\mu}, \tilde{\beta})$ to a sample $\mathbf{y}$ is defined as

$$
\begin{aligned}
& \operatorname{rdepth}((\tilde{\mu}, \tilde{\beta}), \mathbf{y})=\min _{-m \leq i \leq m}\left\{\min \left\{L^{+}(i)+R^{-}(i), R^{+}(i)+L^{-}(i)\right\}\right\} \\
& \text { with } L^{+}(i)=L_{\tilde{\mu}, \tilde{\beta}}^{+}(i)=\#\left\{j \in\{-m, \ldots, i\}: r_{j}(\tilde{\mu}, \tilde{\beta}) \geq 0\right\} \\
& \text { and } R^{-}(i)=R_{\tilde{\mu}, \tilde{\beta}}^{-}(i)=\#\left\{j \in\{i+1, \ldots, m\}: r_{j}(\tilde{\mu}, \tilde{\beta})<0\right\} .
\end{aligned}
$$

$L^{-}(i)$ and $R^{+}(i)$ are defined analogously.

Applying such regression functionals, we estimate the level of the signal and its slope in the centre of the current time window, as in Davies, Fried and Gather (2004). This implies a delay of $m$ time units for the current estimation. As we are rather interested in the level at the most recent time point, which is at the end of the window, we investigate the behaviour of the online estimates defined as $\tilde{\mu}^{\text {online }}=\tilde{\mu}+\tilde{\beta} m$.

\subsection{Algorithms and Computational Speed}

We use update algorithms for all estimates (Bernholt 2004), which prevents calculating the new value for each time window from scratch and thus enhances the computational speed.

The algorithms for $L M S$ and LTS regression are based on the results of Edelsbrunner and Souvaine (1990). The repeated median algorithm is described in detail by Bernholt and Fried (2003), and the deepest regression estimates are computed by an update algorithm based on results from van Kreveld, Mitchell, Rousseeuw, Sharir, Snoeyink and Speckmann (1999). This algorithm does not take the average over all deepest regression fits, if there are several, but chooses one of the deepest fits at random which increases the speed of computation but might lead to some loss of efficiency.

Table 1 shows the computational complexities of the resulting update algorithms. However, these values only reflect asymptotic behaviour. Therefore, 


\begin{tabular}{lcccc}
\hline & $L M S$ & $L T S$ & $R M$ & $D R$ \\
\hline time & $O\left(n^{2}\right)$ & $O\left(n^{2}\right)$ & $O(n)$ & $O\left(n \log ^{2} n\right)$ \\
memory space & $O\left(n^{2}\right)$ & $O\left(n^{2}\right)$ & $O\left(n^{2}\right)$ & $O(n)$ \\
\hline
\end{tabular}

Table 1: Computational complexity of the considered algorithms.

\begin{tabular}{lrrrr}
\hline & LMS & LTS & $R M$ & DR \\
\hline$n=21$ & 0.161 & 0.161 & 0.035 & 0.747 \\
$n=31$ & 0.323 & 0.324 & 0.049 & 0.956 \\
\hline
\end{tabular}

Table 2: Mean computation time of 10000 updates in msec.

Table 2 shows the mean time needed for an update in milliseconds for small sample sizes, measured on a PC with Pentium IV processor with $2.4 \mathrm{GHz}$ and 512 MB memory.

It turns out that, when using these update algorithms, the repeated median is by far the fastest method for the considered sample sizes. In contrast to its low asymptotic computation time, an update of the $D R$ estimate takes about 20 times longer than that of the repeated median. The algorithms for $L M S$ and $L T S$ are faster than that for $D R$ for the small sample sizes considered here; the smaller asymptotic computation time of the latter seems to need considerable sample sizes to become dominant.

\subsection{Breakdown and Exact Fit}

In case of normal errors, least squares is the most efficient regression method. However, least squares regression can be strongly influenced by a single outlier, resulting in a finite sample replacement breakdown point of $1 / n$. Since medical data can contain several outliers within short time spans, we prefer robust methods which show stable results and small bias even for a high percentage of contamination, preferably combined with satisfactory efficiency in periods without measurement problems and artefacts.

$L M S, R M$, and $L T S$ (with $h=\lfloor n / 2\rfloor+1$ ) possess a finite sample replacement breakdown point of $\lfloor n / 2\rfloor / n \approx 50 \%$ which is the highest possible value for a 
regression equivariant functional (Rousseeuw and Leroy 1987). Rousseeuw and Hubert (1999) show that deepest regression has a breakdown point of at least about one third in any case. This raises the question if its breakdown is larger in case of a fixed design, as it is here at hand. For example, the $L_{1}$ breakdown point is $1 / n$ if contamination in the explanatory variable is allowed, while it increases to about $29.3 \%$ in case of an equally-spaced design. However, below we will provide evidence that even in this case deepest regression only guarantees protection against up to one third contaminated observations in the sample.

Therefore, we first regard the exact fit property: Data from intensive care often contain repeated values as the measurements are on a discrete scale, and the patient's physiological parameters can stay steady for some time. In such situations the exact fit property is informative.

A regression functional $T: \mathbb{R}^{n} \rightarrow \mathbb{R}^{2}$ possesses the exact fit property if for some fit $(\tilde{\mu}, \tilde{\beta})$ and $k \in\{0,1, \ldots,\lceil n / 2\rceil-1\}$ the following is satisfied: Whenever $y_{i}=\tilde{\mu}+\tilde{\beta} i$ fits at least $n-k$ of the $n$ observations exactly, then $T=(\tilde{\mu}, \tilde{\beta})$ whatever the other $k$ observations are. Roughly spoken: if the majority of the data lies on a straight line, the solution of the functional $T$ will be exactly this line (Rousseeuw and Leroy 1987, p. 122).

The smallest possible fraction of contamination which can cause a regression functional $T$ to deviate from $(\tilde{\mu}, \tilde{\beta})$ is called the exact fit point: consider a sample $\mathbf{y}_{n}$ of size $n$ such that $y_{i}=\tilde{\mu}+\tilde{\beta} i$ for all $i$, and let $\mathbf{y}_{k, n}$ be a sample where $k$ out of the $n$ observations of $\mathbf{y}_{n}$ are replaced by arbitrary values. Then, the exact fit point of $T$ is defined as

$$
\delta_{n}^{*}\left(T, \mathbf{y}_{n}\right)=\min _{k}\left\{\frac{k}{n} \mid \text { there exists a sample } \mathbf{y}_{k, n} \text { such that } T\left(\mathbf{y}_{k, n}\right) \neq(\tilde{\mu}, \tilde{\beta})\right\} \text {. }
$$

For regression and scale equivariant functionals as considered here, this value gives an upper bound for the finite sample replacement breakdown point $\varepsilon_{n}^{*}$ (Rousseeuw and Leroy 1987, pp. 122-124), i.e.

$$
\varepsilon_{n}^{*}\left(T, \mathbf{y}_{n}\right) \leq \delta_{n}^{*}\left(T, \mathbf{y}_{n}\right)
$$

The exact fit point for $L M S$ and $L T S$ is $\frac{\lceil n / 2\rceil}{n}$ (Rousseeuw and Leroy 1987, Section 3.4). For $R M$ one less observation is needed to pull the fit away 


\begin{tabular}{r|rrrrrrrrrrrr}
$n$ & 5 & 7 & 9 & 11 & 13 & 15 & 17 & 19 & 21 & 23 & 25 & 27 \\
\hline$k$ & 2 & 2 & 3 & 4 & 4 & 5 & 6 & 6 & 7 & 8 & 8 & 9 \\
$n$ & 29 & 31 & 33 & 35 & 37 & 39 & 41 & 43 & 45 & 47 & 49 & 51 \\
\hline$k$ & 10 & 10 & 11 & 12 & 12 & 13 & 14 & 14 & 15 & 16 & 16 & 17
\end{tabular}

Table 3: Upper bound for the exact fit point $k / n$ of the deepest regression functional for selected sample sizes $n$.

from the line in case of a sample of odd size, because its slope component is calculated by taking sets of two observations. Hence, its exact fit point is $\frac{\lfloor n / 2\rfloor}{n}$ which is equal to its breakdown point.

For deepest regression an upper bound for the exact fit point can be derived as follows: consider a sample $\mathbf{y}_{n, k}$ of size $n$ where $n-k$ observations lie on a straight line $l_{0}: y_{j}=\mu_{0}+\beta_{0} j, j=-m, \ldots, m$. The exact fit point $\delta_{n}^{*}$ equals the smallest fraction $k / n$ of values not lying on $l_{0}$ such that the deepest regression fit departs from the line $l_{0}$. This means we are searching for a number $k$ with $T_{D R}\left(\mathbf{y}_{n, k-1}\right)=\left(\mu_{0}, \beta_{0}\right)$ and $T_{D R}\left(\mathbf{y}_{n, k}\right) \neq\left(\mu_{0}, \beta_{0}\right)$.

W.l.o.g. we assume $\mu_{0}=0$ and $\beta_{0}=0$. Furthermore, we take the first $n-k$ observations to lie on the line $l_{0}$, i.e. we have $y_{j}=0$ for $j=-m, \ldots, m-k$, and we put the remaining $k$ observations on another line $l_{1}: y_{j}=\mu_{1}+\beta_{1} j$ for $j=m-k+1, \ldots, m$, with $\mu_{1}=-\frac{n+1}{2}$ and $\beta_{1}=1 \neq \beta_{0}$. This guarantees that $l_{1}$ has a regression depth of at least $k$, because at least $k$ observations lie on $l_{1}$. Also, the residuals of these observations have the same (positive) sign with respect to $l_{0}$. In this way, the fit of $l_{0}$ to the full sample $\mathbf{y}_{n, k}$ is worsened with increasing $k$. Table 3 gives the smallest number $k$ of non-zero observations which, in this configuration, forces the deepest regression estimate away from $(0,0)$ for small to moderate sample sizes.

In this particular data situation and for the considered sample sizes, we see that the departure of $\left\lfloor\frac{n+1}{3}\right\rfloor$ observations from $l_{0}$ can cause the deepest regression fit to do so too. 
Hence, we can conclude that the smallest $k$ with $T_{D R}\left(\mathbf{y}_{n, k}\right) \neq(\mu, \beta)$ is at most $\left\lfloor\frac{n+1}{3}\right\rfloor$ and thus

$$
\delta_{n}^{*}\left(T_{D R}, \mathbf{y}\right) \leq \frac{1}{n} \cdot\left\lfloor\frac{n+1}{3}\right\rfloor
$$

Rousseeuw and Hubert (1999) show that the breakdown point of the $T_{D R}$ at any data set is at least one third:

$$
\varepsilon_{n}^{*}\left(T_{D R}, \mathbf{y}\right) \geq \frac{1}{n}\left(\left\lceil\frac{n}{3}\right\rceil-1\right) \approx \frac{1}{3} .
$$

Thus,

$$
\frac{1}{n}\left(\left\lceil\frac{n}{3}\right\rceil-1\right) \leq \varepsilon_{n}^{*}\left(T_{D R}, \mathbf{y}\right) \leq \delta_{n}^{*}\left(T_{D R}, \mathbf{y}\right) \leq \frac{1}{n} \cdot\left\lfloor\frac{n+1}{3}\right\rfloor .
$$

This leads to the claim that, even in case of an equally-spaced design, the breakdown point of the $D R$ functional equals $1 / 3$.

\section{Monte Carlo Study}

In the following, we compare the performance of the online estimates $\tilde{\mu}^{\text {online }}=$ $\tilde{\mu}+\tilde{\beta} m$ in different data situations. In particular, we consider scenarios which are of importance in the online monitoring context. The performance of the estimates will be judged by their standard deviation, bias and root mean squared error. For comparison, we also include results for least squares $(L S)$ regression. Data are generated from the simple linear model

$$
Y_{i}=\mu+\beta i+\varepsilon_{i}, i=-m, \ldots, m \text {. }
$$

where for $\varepsilon_{i}$ we consider

- normal errors,

- heavy tailed errors,

- skewed errors,

- normal errors with additive outliers at random time points,

- normal errors with subsequent additive outliers.

We set $\mu=\beta=0$ w.l.o.g., since all methods considered here are regression equivariant, and set the error variance to one w.l.o.g. because of the scale equivariance. In each case $S=10000$ independent samples are generated. 
On the one hand, the assumption of a linear trend within each time window becomes less reliable if a large window width is chosen: in this case, even a small bias in the estimation for the window centre can cause a considerable bias of the online estimates as these are based on linear extrapolation. On the other hand, a large window width stands for smaller variability and produces smoother estimates. As a compromise, a choice of $m=10$ or $m=15$ is considered acceptable for the physiological data we have in mind, leading to window widths of $n=21$ or $n=31$ respectively, with the time units being minutes.

\subsection{Standard Normal Errors}

In the ideal situation of normal errors all methods yield unbiased results, due to the symmetry of the underlying error distribution.

Repeated median and deepest regression do not perform much worse than least squares $(L S)$ regression whilst the $L M S$ and $L T S$ estimates spread much further (cf. Table 4). The similar behaviour of LMS and LTS can be explained by the fact that both pick about $50 \%$ of the observations which can be optimally described by a straight line, without restrictions for symmetry, while $R M$ and $D R$ seek for a balanced fit.

As a result, the $L M S$ and $L T S$ online estimates are only slightly more than $20 \%$ as efficient as $L S$, while for $D R$ we have about $61 \%$, and for $R M$ approximately $70 \%$ efficiency. This is consistent with previous research, and the results here even reflect the fact that for small samples $L M S$ regression is slightly more efficient than LTS regression (Rousseeuw and Leroy 1987).

\subsection{Heavy Tails and Skewness}

As real data sets may contain large aberrant values, the normal distribution is often not appropriate to model the error term. Therefore, we examine errors from a re-scaled $t$-distribution with three degrees of freedom and unit variance as well as errors from a shifted lognormal distribution with zero median and unit variance. 


\begin{tabular}{c|rrrrrr}
\hline & & $L M S$ & $L T S$ & $R M$ & $D R$ & $L S$ \\
\hline standard normal & $n=21$ & 0.875 & 0.887 & 0.500 & 0.533 & 0.420 \\
errors & $n=31$ & 0.767 & 0.785 & 0.422 & 0.450 & 0.352 \\
\hline heavy tailed & $n=21$ & 0.544 & 0.551 & 0.345 & 0.354 & 0.413 \\
errors & $n=31$ & 0.450 & 0.455 & 0.279 & 0.287 & 0.342 \\
\hline skewed & $n=21$ & 0.489 & 0.495 & 0.353 & 0.384 & 0.429 \\
errors & $n=31$ & 0.389 & 0.399 & 0.285 & 0.317 & 0.350 \\
\hline
\end{tabular}

Table 4: Standard deviations for the estimates at standard normal, re-scaled $t_{3}$ distributed and re-scaled lognormal data.

At the $t_{3}$-distribution, all methods yield unbiased results because of symmetry. Again, the results for $L M S$ and $L T S$ regression are similar, like those for $R M$ and $D R$. The standard deviations (cf. Table 4) show that compared to the standard normal situation the variability has decreased for all robust methods, while for least squares it remains about the same since its standard deviation only depends on the error variance.

A larger window width causes less variability, but the proportions of the outcomes from the different methods stay approximately the same for different window widths. The LMS and LTS standard deviations are about $60 \%$ the size of their values in the standard normal case, but nevertheless they are still outperformed by $L S$. This is not true for $R M$ and $D R$, having standard deviations about $66 \%$ of their former size, with repeated median regression showing the smallest variability here.

Figure 1 shows boxplots of the results for the online estimates at lognormal errors with a window width of $n=31$. The black line in the box denotes the median, the grey line the arithmetic mean.

The figure clearly shows systematic differences among the considered methods. Rousseeuw, Van Aelst and Hubert (1999) point out that LMS and LTS are 'mode-seeking' in contrast to the 'median-like' behaviour of deepest regression and, as we want to add, the repeated median. Indeed, the least median of squares and least trimmed squares estimates lie mainly between the mode and the median of the underlying error distribution, while repeated median and 


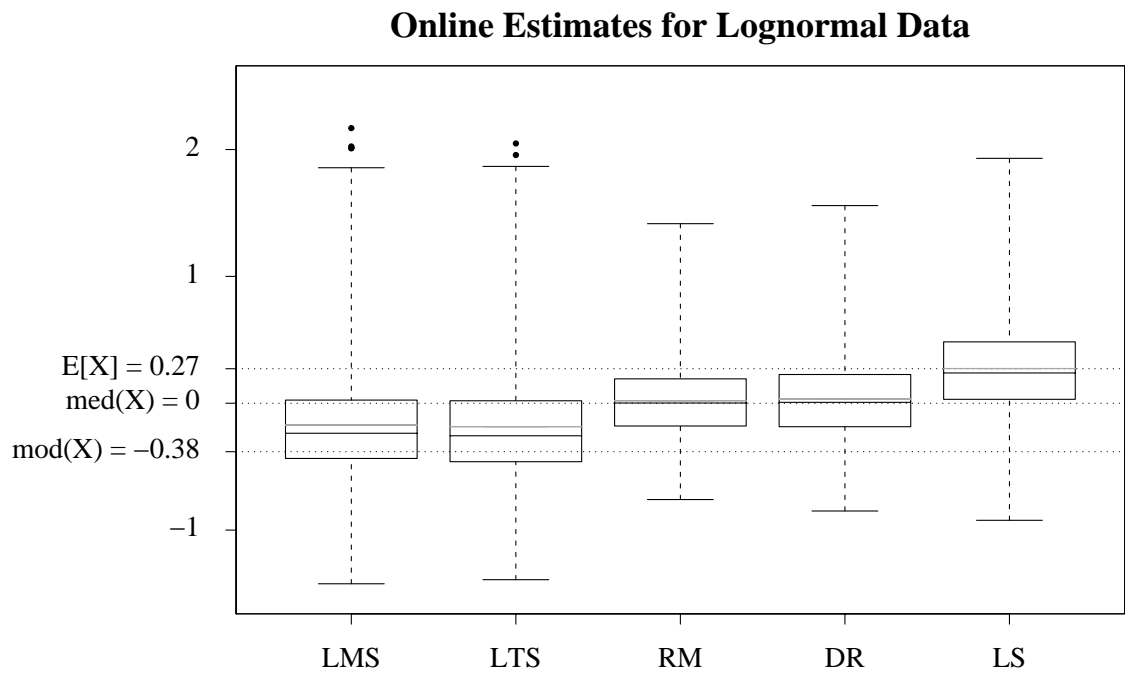

Figure 1: Boxplots of the simulation results for the window width $n=31$.

deepest regression yield results centred at the median and least squares at the expectation.

Since the methods apparently estimate different quantities, an examination of bias is not sensible. Thus, we will only regard variability (cf. Table 4).

The $R M$ and $D R$ standard deviations are only about $70 \%$ that for the standard normal situation, and for $L M S$ and $L T S$ they are only about half as large. Comparing the results of the robust methods to least squares we see that the $R M$ standard deviation is only slightly more than $80 \%$ as large as the corresponding least squares value, while the $D R$ standard deviation is approximately $90 \%$ as large. $L M S$ and $L T S$ on the other hand perform again worse than least squares where $L T S$ shows a little more variability than $L M S$. Hence, again the repeated median provides the best results.

\subsection{Additive Outliers}

In intensive care, data suffer from a broad variety of perturbations, either caused by medical reasons or by external sources such as a loose cable. As these disturbances often produce similar deviations at several time points, we investigate the influence of additive outliers with same sign and size. 
Standard Deviation
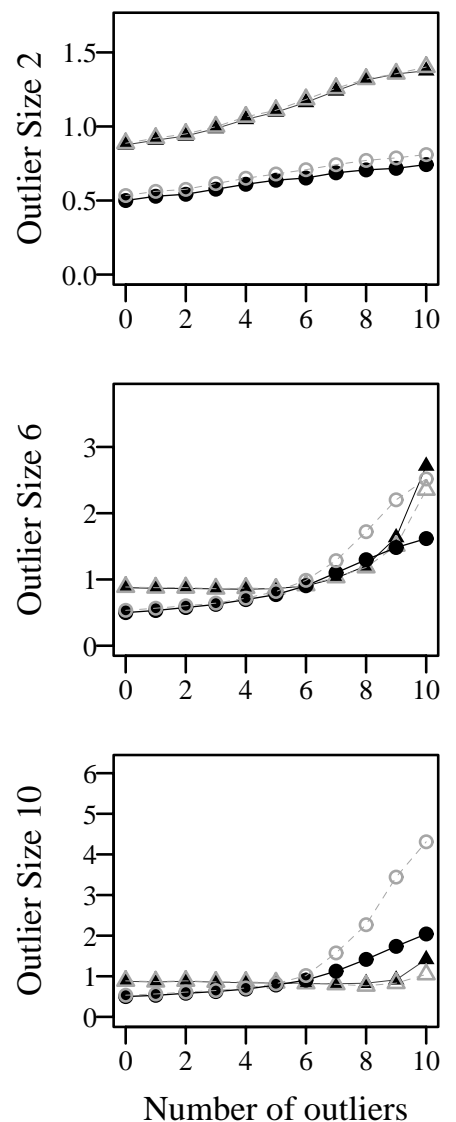

Bias
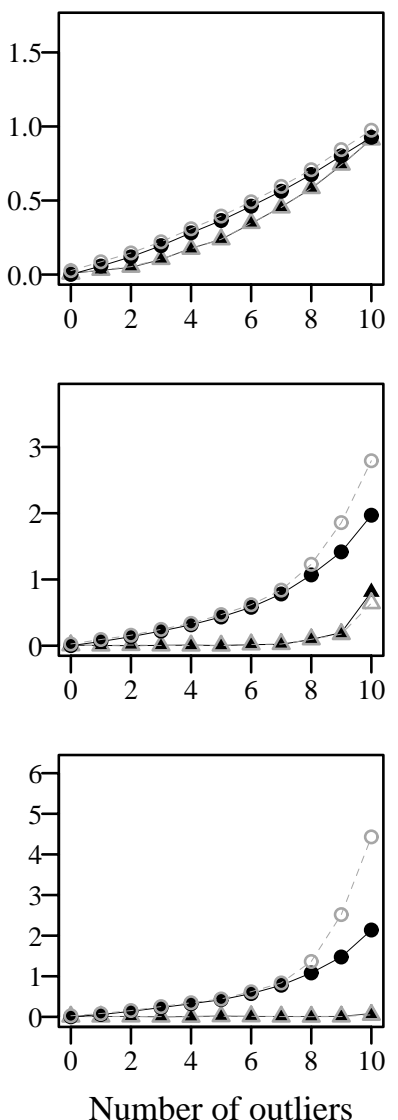

RMSE
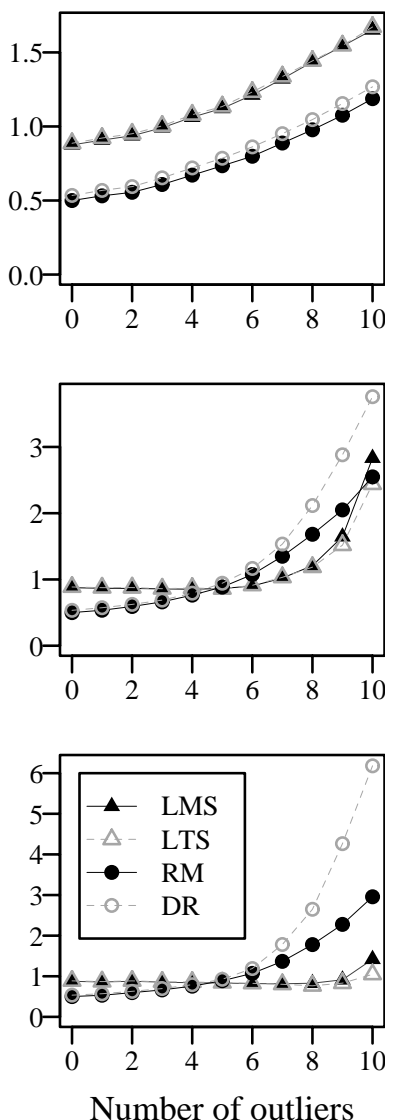

Figure 2: Standard deviation, bias and root mean squared error ( $R M S E$ ) for the online estimates at standard normal data with additive outliers at random time points.

We generate samples from a standard normal model and add a value $a \in$ $\{2,4,6,8,10\}$ to an increasing number $k \in\{1, \ldots, 10\}$ of observations chosen at random from the sample. Negative additive outliers would yield analogous results. For the sake of brevity we only consider the sample size $n=21$.

Outliers at random time points do not cause a bias for the slope, but for the level estimation, which also affects the online estimates. Figure 2 shows standard deviation, bias and root mean squared error ( $R M S E)$ of the online estimates for outliers of size 2, 6 and 10. Results for outlier sizes of 4 and 8 lie in between. 
Again, the similarity of the $R M$ and $D R$ outcomes shows up clearly, and the differences in the results between $L M S$ and LTS regression are negligible. $L T S$ is only slightly less variable than $L M S$ for a large number of $9-10$ outliers. We also see that $L M S$ and $L T S$ are more heavily affected by smaller outliers than by larger ones.

Comparing repeated median and deepest regression, $R M$ is preferable here as it yields a smaller standard deviation and bias for all considered numbers and sizes of outliers. However, this advantage is only significant in case of seven or more outliers in accordance with the lower breakdown point of deepest regression.

Overall, $L M S$ and $L T S$ perform best in terms of bias although with respect to the $R M S E$ they only outperform the other methods in case of many large outliers. For small outliers or a small to moderate number of outliers the repeated median should be preferred as it has the smallest $R M S E$.

\subsection{Outlying Sequences}

For online monitoring it is of special importance to track sudden jumps in the signal because this may point at an abrupt change of the patient's state. Looking at single time windows such a level shift is indicated by a patch of outlying values of the same size and sign at the end of a time window.

We simulate such situations by generating positive additive outliers of the same size as in the previous subsection - only that now the value $a \in\{2,4,6,8,10\}$ is added to $k \in\{1, \ldots, 10\}$ subsequent values at the end of the time window. Again, only the case $n=21$ is investigated.

As the online estimates approximate the level at the end of the window, a small bias w.r.t. level in the centre of the time window is not necessarily what we aim at: in intensive care monitoring, as a medical rule of thumb a sequence of five or more largely deviating values is assumed to indicate a shift whereas a smaller number is typically regarded as series of outliers (Imhoff, Bauer, Gather and Fried 2003). Hence, a method performs well if it maintains the central level in case of a few subsequent outliers but jumps up to the level 
Standard Deviation
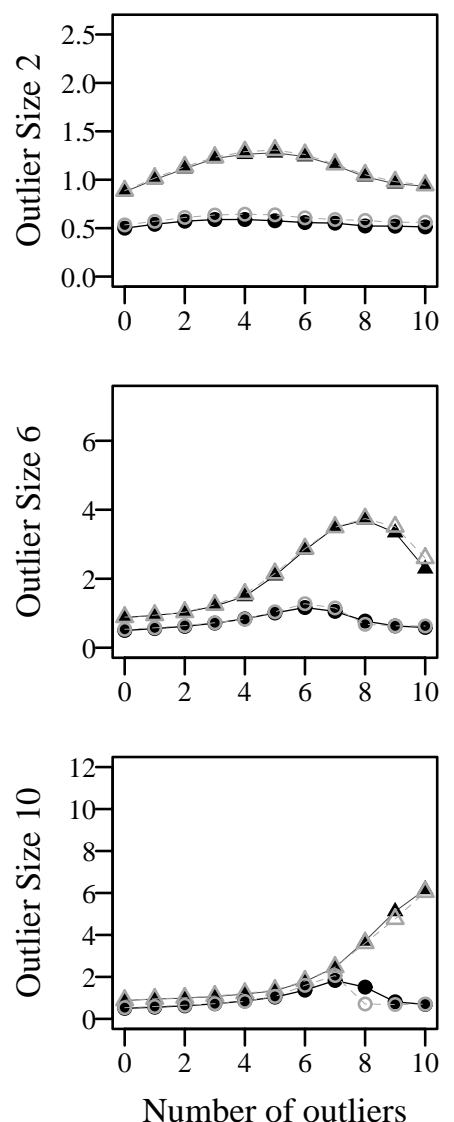

Bias
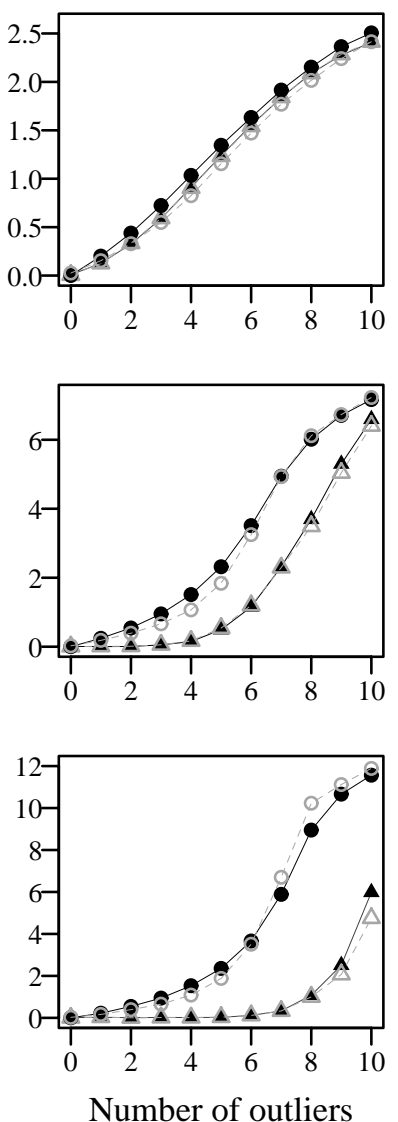

RMSE
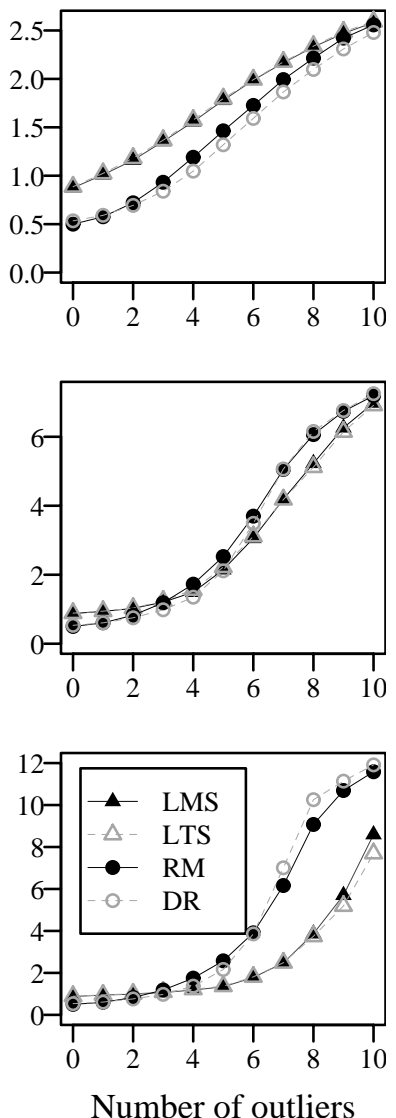

Figure 3: Standard deviation, bias and root mean squared error $(R M S E)$ for the online estimates at standard normal data with additive outliers, occurring subsequently at the end of the time window.

of these largely deviant observations when their number is five or more - to estimate the new (higher) level rather than the former (lower) one in the centre of the window.

Again, Figure 3 shows standard deviation, bias and $R M S E$ only for outlier sizes 2, 6 and 10 as the results for the sizes 4 and 8 lie in between.

No method shows exactly the bias behaviour described above, although for medium-sized to large outliers the LMS and LTS bias curves remain constantly low for a smaller number of outliers and then show a sudden drastic 
increase. However, the number of outliers which is necessary to make the $L M S$ or $L T S$ bias increase is the larger, the larger the size of the outliers is. In other words: the LMS and LTS online estimates follow a large level shift with a considerable delay, in contrast to the estimates obtained by these methods in the window centre, see Davies et al. (2004). On the other hand, the $R M$ and $D R$ estimates typically smear a moderately large shift.

Also, one can derive from Figure 3 that the standard deviations of $R M$ and $D R$ are always smaller than those of $L M S$ and LTS regression, and further that they stay almost constant. Again, the difference between $L M S$ and $L T S$, and between $R M$ and $D R$ is negligible, both with respect to bias and variability, in spite of the different breakdown points of the latter.

\section{Application to Time Series}

In this section, we analyse the stability of the estimates as well as their ability to track trends, slope changes and sudden level shifts by applying them to a simulated and to a real time series. In both cases we use a window width of $n=21$ observations.

The simulated time series is 250 time units long and consists of a signal containing constant as well as trend periods and a level shift, plus additive standard normal noise. $10 \%$ of the observations are replaced by positive additive outliers of size 6, which are bundled in patches of four subsequent outliers (twice), three outliers (twice), two outliers (three times), and single outliers (five times). The starting point of each sequence is chosen at random.

Figure 4 shows the online estimates and the underlying signal for the simulated times series. All methods trace the trends and the slope changes. Also, the similarity of the results from $L M S$ and $L T S$ regression as well as from repeated median and deepest regression shows up clearly.

$R M$ and $D R$ yield more stable results than $L M S$ and $L T S$, and they are less influenced by values deviating moderately from the underlying signal, e.g. see the results around time points $50-60$ and around time point 150 . 


\section{Time Series with Standard Normal Noise and $10 \%$ Positive Outliers}
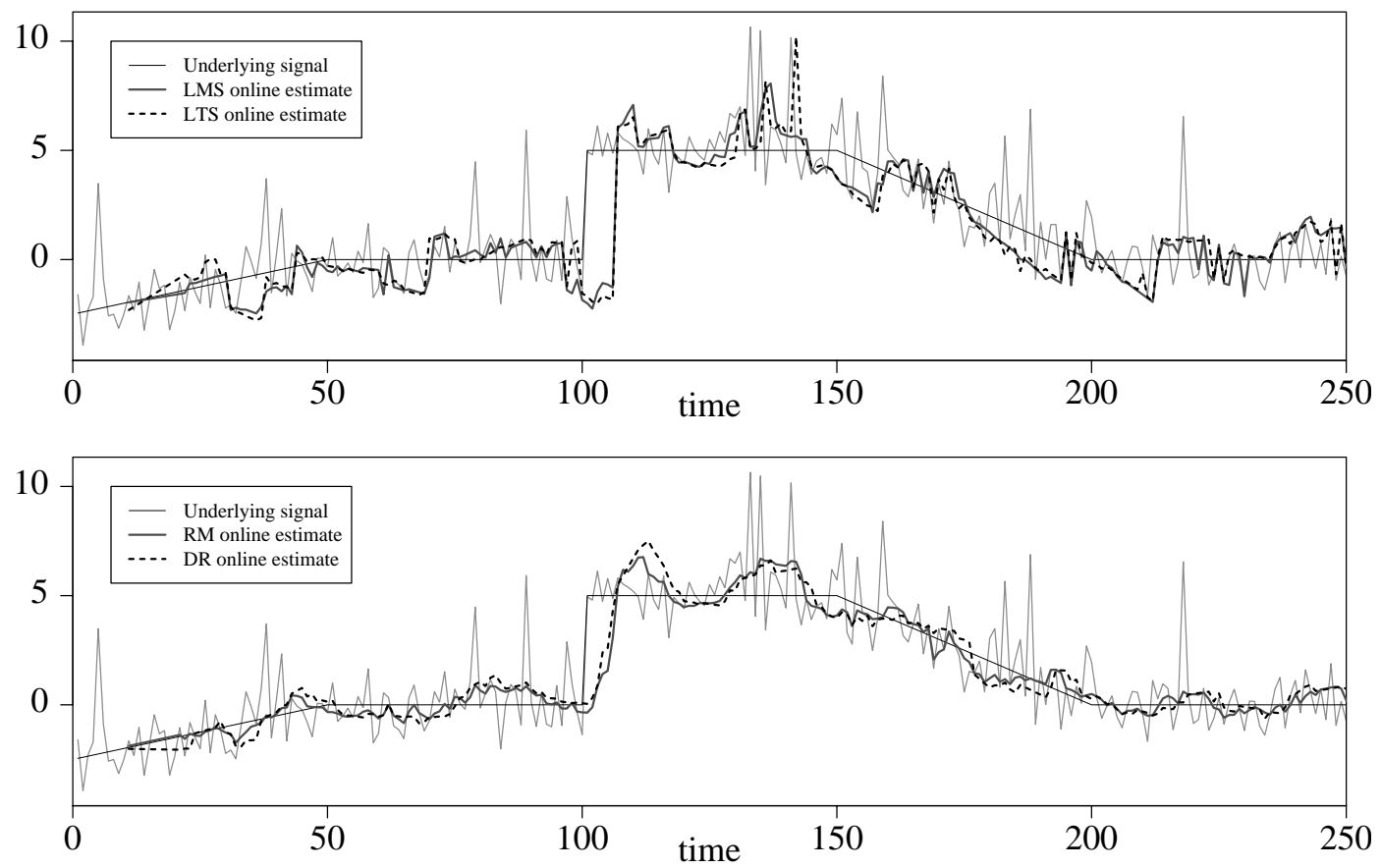

Figure 4: Online estimates based on windows of size $n=21$.

As the online estimates are based on a linear extrapolation of the level estimates for the centre of the time window, the LMS and LTS estimates continue the pre-level-shift trend until some time points after the level shift. This is due to their small bias with respect to the 'old' level before the shift.

Repeated median and deepest regression trace the level shift with a shorter delay than LMS or LTS, but they do not capture the abruptness of the jump. Also, the $R M$ and $D R$ estimates are closer to the signal around the times of a slope change - especially around the times 150 and 200. After the transition to the 'new' level, subsequent to the shift, all methods overestimate the signal, due to the strongly positive slope estimates around the shift. This is a well-known phenomenon when using a local linear fit, see e.g. Einbeck and Kauermann (2003).

Finally, we apply the methods to a medical time series of length 250, representing the mean pulmonary artery blood pressure of an intensive care patient. 


\section{Time Series of a Mean Pulmonary Artery Blood Pressure}
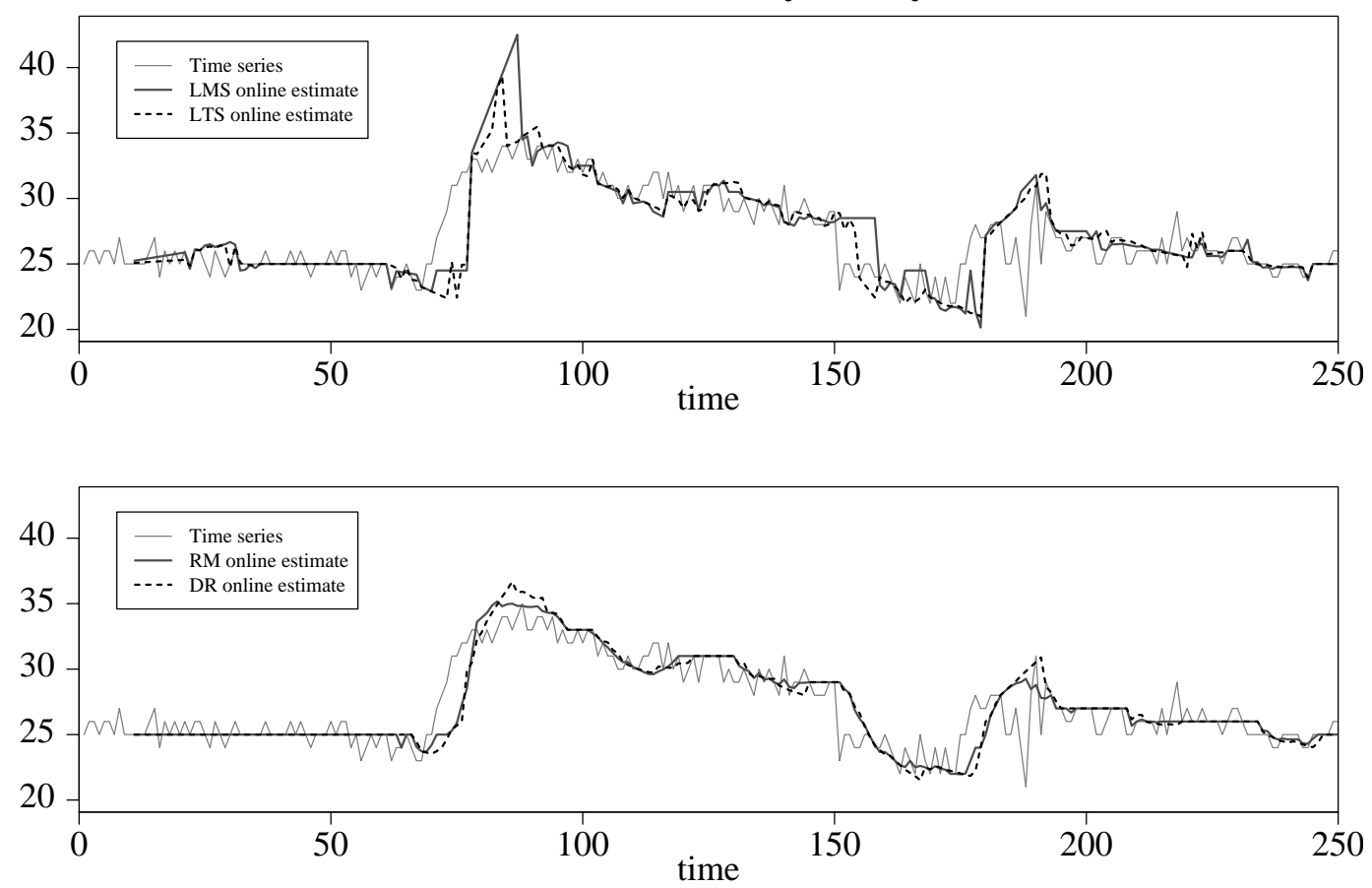

Figure 5: Online estimates based on windows of size $n=21$.

Figure 5 shows that $R M$ and $D R$ yield much more stable results while $L M S$ and LTS are affected by moderate variation in the data.

The $R M$ and $D R$ method trace the level shift around time point 70 better than $L M S$ or LTS regression. Also, LMS and LTS overestimate the level right after the shift by far more drastically. However, they capture the abruptness of the shifts better (e.g. around the times 150 and 175) while $R M$ and $D R$ smear them. Here, the analyst must decide whether it is better to get a 'smeared' transition from one level to the other, or to catch the suddenness of the jump with some time delay.

Both examples show the superiority of repeated median and deepest regression in terms of stability. Also, the repeated median does not overestimate the signal after a shift as much as deepest regression. 


\section{Conclusions}

All of the considered methods follow trends and slope changes and trace level shifts quite well. The differences in the outcomes from least median of squares and least trimmed squares regression are negligible while repeated median and deepest regression also show very similar results.

For symmetric, unimodal errors all methods provide unbiased estimates of the median and the mode, which are identical in this case; in case of unimodal, but skewed errors, the LMS and LTS estimates lie somewhere in between the median and the mode while $R M$ and $D R$ estimate the median.

$L M S$ and $L T S$ are less biased than $R M$ and $D R$ in the presence of many large outliers. However, as explained in Section 3.4, in case of a level shift a small bias does not mean better performance of the online estimates. Although $R M$ and $D R$ smear a shift somewhat, these methods still might be preferred because $L M S$ and $L T S$ follow a shift with a longer delay - especially if the shift is large. In spite of the claim that deepest regression is particularly appropriate for skewed errors due to its construction, the repeated median performed even better for lognormal errors.

Further, repeated median and deepest regression yield a more stable signal extraction; and the LMS and LTS estimates are stronger influenced by small or medium-sized outliers.

Summarising, repeated median and deepest regression outperform $L M S$ and LTS regression w.r.t. online signal extraction without delay. Repeated median regression yields the best results in most respects: among these robust methods, $R M$ is the least variable in most of the considered situations; it gives stable estimations in the applications and also, it is computationally the fastest.

Acknowledgement: We gratefully acknowledge the financial support of the Deutsche Forschungsgemeinschaft (SFB 475: 'Reduction of Complexity for Multivariate Data Structures'). 


\section{References}

Bernholt, T. (2004). Update Algorithms for the Repeated Median, LMS, LTS and Deepest Regression, Personal Communication.

Bernholt, T. and Fried, R. (2003). Computing the Update of the Repeated Median Regression Line in Linear Time, Inf. Process. Lett. 88 (1), 111117.

Davies, P.L., Fried, R. and Gather, U. (2004). Robust Signal Extraction for On-line Monitoring Data, J. Stat. Plann. Inference 122 (1-2), 65-78.

Edelsbrunner, H. and Souvaine, D.L. (1990). Computing Least Median of Squares Regression Lines and Guided Topological Sweep, J. Am. Stat. Assoc. 85, No. 409, 115-119.

Einbeck, J. and Kauermann, G. (2003). Online Monitoring with Local Smoothing Methods and Adaptive Ridging, J. Statist. Comput. Simul. 73, 913929.

Imhoff, M., Bauer, M., Gather, U. and Fried, R. (2002). Pattern Detection in Intensive Care Monitoring Time Series with Autoregressive Models: Influence of the AR-Model Order, Biom. J. 44, 746-761.

Rousseeuw, P.J. (1983). Multivariate Estimation with High Breakdown Point, in W. Grossmann, G. Pflug, I. Vincze, W. Wertz (eds.) Proceedings of the 4th Pannonian Symposium on Mathematical Statistics and Probability, Vol. B, D. Reidel Publishing Company, Dordrecht (The Netherlands).

Rousseeuw, P.J. (1984). Least Median of Squares Regression, J. Am. Stat. Assoc. 79, No. 388, 871-880.

Rousseeuw, P.J. and Hubert, M. (1999). Regression Depth, J. Am. Stat. Assoc. 94, No. 446, 388-402.

Rousseeuw, P.J. and Leroy, A.M. (1987). Robust Regression and Outlier Detection, Wiley, New York (USA). 
Rousseeuw, P.J., Van Aelst, S. and Hubert, M. (1999). Rejoinder to 'Regression Depth', J. Am. Stat. Assoc. 94, No. 446, 419-433.

Siegel, A.F. (1982). Robust Regression Using Repeated Medians, Biometrika 69, 242-244.

Van Kreveld, M., Mitchell, J.S.B., Rousseeuw, P.J., Sharir, M., Snoeyink, J. and Speckmann, B. (1999). Efficient Algorithms for Maximum Regression Depth, Proceedings of the 15th Annual ACM Symposium of Computational Geometry, ACM Press, New York (NJ), 31-40. 